\title{
Large time behavior of Euler-Poisson system for semiconductor
}

\author{
Feimin Huang ${ }^{1}$, Ronghua $\mathrm{Pan}^{2 \dagger}$, Huimin $\mathbf{Y u}^{1 \ddagger}$ \\ ${ }^{1}$ Institute of Applied Mathematics, AMSS, Academia Sinica, Beijing 100080, China \\ ${ }^{2}$ School of Mathematics, Georgia Institute of Technology, Atlanta, GA 30332, USA
}

Abstract: In this note, we present a framework for the large time behavior of general uniformly bounded weak entropy solutions to the Cauchy problem of Euler-Poisson system of semiconductor devices. It is shown that the solutions converges to the stationary solutions exponentially in time. No smallness and regularity conditions are assumed.

\section{Introduction}

Consider the following one-dimensional Euler-Poisson system modeling semiconductor devices:

$$
\left\{\begin{array}{l}
n_{t}+J_{x}=0 \\
J_{t}+\left(\frac{J^{2}}{n}+p(n)\right)_{x}=n E-J, \quad x \in \mathbf{R}, t>0, \\
E_{x}=n-b(x) .
\end{array}\right.
$$

Here $n \geq 0, J$, and $E$ denote the electron density, electron current density and the (negative) electric field, respectively. The function $b=b(x)>0$, called doping profile, stands for the density of fixed, positively charged background ions. In this paper, we assume $b(x)$ satisfies

$$
\begin{gathered}
b(x) \in C^{2}(\mathbf{R}), b^{\prime}(x) \in L^{1}(\mathbf{R}) \cap H^{1}(\mathbf{R}), \\
\lim _{x \rightarrow \pm \infty} b(x)=b^{ \pm}>0, \quad b^{*}=\sup _{x \in \mathbf{R}} b(x) \geq \inf _{x \in \mathbf{R}} b(x)=b_{*}>0 .
\end{gathered}
$$

\footnotetext{
*Email address: fhuang@amt.ac.cn,

$\dagger$ Email address: panrh@math.gatech.edu,

${ }^{\ddagger}$ Email address: hmyu@amss.ac.cn,
} 
We also assume the pressure $p$ satisfies the $\gamma$-law: $p(n)=n^{\gamma}(\gamma \geq 1)$. Several physical constants have been set to unity for the simplicity of presentation. Such a system, replacing the most commonly used drift-diffusion model for charged carriers, is valid in the region occupied by the semiconductor. We refer to [14] for background on modeling and analysis.

This system is supplemented with a condition at $x=-\infty$ for the electric field

$$
\lim _{x \rightarrow-\infty} E(x, t)=E^{-}, \quad \text { for } \quad \text { a.e. } \quad t \in[0,+\infty),
$$

and the initial conditions

$$
n(x, 0)=n_{0}(x), \quad J(x, 0)=J_{0}(x)
$$

such that

$$
\lim _{x \rightarrow \pm \infty} n_{0}(x)=b^{ \pm}>0, \quad \lim _{x \rightarrow \pm \infty} J_{0}(x)=\bar{J}, \quad b_{-} E^{-}=\bar{J} .
$$

Due to the relaxation mechanism, smooth solutions exist globally in time if the initial data is chosen from the so-called sub-critical region; see [1] and [17]. However, when data is out of that region, the solutions break down in finite time, c.f. [2]. The global existence of weak entropy solutions are proven in [13], [16] and [18]. Concerning with the large time behavior of solutions, we refer to [10], [11] for small smooth solutions, and to [9] for piecewise smooth solutions. There were also some results concerning the initial boundary value problems, we refer the readers to [8], [12] and [4] and the references therein.

In this paper, we will focus on a framework on large time asymptotic behavior that applies to any uniformly bounded entropy weak solutions. The entropy and entropy flux pair we will use here are the physical ones defined as following:

$$
\begin{array}{lll}
\eta_{e}=\frac{J^{2}}{2 n}+\frac{n^{\gamma}}{\gamma-1}, & q_{e}=\frac{J^{3}}{2 n^{2}}+\frac{\gamma}{\gamma-1} n^{\gamma-1} J, & \gamma>1 ; \\
\eta_{e}=\frac{J^{2}}{2 n}+n \ln n, & q_{e}=\frac{J^{3}}{2 n^{2}}+(\ln n+1) J, & \gamma=1 .
\end{array}
$$

We now define the concept of entropy weak solutions.

Definition 1: The bounded measurable function $(n, J, E)(x, t)$ is said to be an entropy weak solution of problem (1), (3)-(5), if it satisfies the system 
(1) in the distributional sense, verifies the initial and limiting restrictions (3)-(5), and the following entropy inequality

$$
\eta_{e t}+q_{e x}+\frac{J}{n}(J-n E) \leq 0,
$$

holds in the distributional sense.

Throughout this paper, we assume

H1): Assume that $(n, J, E)(x, t)$ is any globally defined weak entropy solutions which satisfies

$$
0 \leq n(x, t) \leq C_{0} .
$$

Under this assumption, we will prove that the entropy weak solution defined above converges exponentially fast toward the corresponding stationary states if the background current $\bar{J}$ has small amplitude. In section 2 , we will study the stationary states in subsonic region. Our main result will be established in section 3. Some remarks are collected in section 4 .

\section{Stationary states}

Again, due to the relaxation mechanism in the current equation of (1), we expect that all the solutions of our problem converge to the solutions of the following stationary problem,

$$
\left\{\begin{array}{l}
\tilde{J}_{x}=0, \\
\left(\frac{\tilde{J}^{2}}{\tilde{n}}+p(\tilde{n})\right)_{x}=\tilde{n} \tilde{E}-\tilde{J}, \\
\tilde{E}_{x}=\tilde{n}-b(x),
\end{array}\right.
$$

under the conditions

$$
\tilde{n}(x)-b(x) \in H^{1}(\mathbf{R}), \quad \tilde{J}=\bar{J}, \quad \tilde{E}(-\infty)=E^{-} .
$$

A straightforward calculation (see [9]) shows that (9)-(10) gives

$$
\left[\left(\frac{p^{\prime}(\tilde{n})}{\tilde{n}}-\frac{\bar{J}^{2}}{\tilde{n}^{3}}\right) \tilde{n}_{x}\right]_{x}+\left(\frac{\bar{J}}{\tilde{n}}\right)_{x}=\tilde{n}-b(x),
$$


which is a second order ODE for $\tilde{n}(x)$. Clearly, the strictly elliptic condition is equivalent to

$$
p^{\prime}(\tilde{n})>\frac{\bar{J}^{2}}{\tilde{n}^{2}}
$$

This is exactly the subsonic condition [3], recalling that $\frac{J}{n}$ represents the particle velocity. We also note that (12) is equivalent to

$$
\tilde{n}>\left(\frac{\bar{J}^{2}}{\gamma}\right)^{\frac{1}{(\gamma+1)}} .
$$

In order to ensure the subsonic condition, we assume that

H2): $b(x)$ and $\bar{J}$ satisfy $b_{*}>\left(\frac{\bar{J}^{2}}{\gamma}\right)^{1 /(\gamma+1)}$.

Under H2), it is proven by [11] (see also [9]) that

Theorem 1: Suppose $b(x)$ satisfies the condition (2). Assume that H2) holds. Then problem (9)-(10) has a unique solution $(\tilde{n}, \bar{J}, \tilde{E})$, such that $b_{ \pm} \tilde{E}( \pm \infty)=\bar{J}$, and

$$
\begin{gathered}
b_{*} \leq \tilde{n}(x) \leq b^{*}, \quad x \in \mathbf{R}, \\
\left|\tilde{n}(x)-b_{ \pm}\right|=O(1) e^{-c_{ \pm}|x|}, \quad \text { as } \quad x \rightarrow \pm \infty, \\
\|\tilde{n}-b\|_{H^{2}}+\sup _{x \in \mathbf{R}}\left(\left|\tilde{n}^{\prime}(x)\right|+\left|\tilde{n}^{\prime \prime}(x)\right|+|\tilde{E}(x)|\right) \leq C_{1},
\end{gathered}
$$

where $C_{1}$ is a positive constant that only depends on $b(x)$, and

$$
c_{ \pm}=\frac{\tilde{E}_{ \pm}}{p^{\prime}\left(b_{ \pm}\right)-\tilde{E}_{ \pm}^{2}} .
$$

We remark that, (14) and H2) together ensure the subsonic condition (13) and then the proof of Theorem 1 is carried out by standard ODE theory. The proof of statement (14) is done through a comparison argument as that in $[10]$. 


\section{Large time behavior}

Now, our aim is to prove the entropy-weak solution of (1), (3)-(5) strongly converges to its stationary solution in $L^{2}(\mathbf{R})$ with exponential decay rate. We set

$$
y=-(E-\tilde{E}) .
$$

Then systems (1) and (9) infer that

$$
y_{x}=-(n-\tilde{n}), \quad y_{t}=J-\bar{J} .
$$

As expected, the entropy inequality (7) will play an important role in our analysis. For this purpose, we introduce

$$
\begin{aligned}
& \eta_{*}=\eta_{e}-\tilde{\eta}_{e}-\nabla \tilde{\eta}_{e}(\vec{v}-\overrightarrow{\tilde{v}}), \\
& q_{*}=q_{e}-\tilde{q}_{e}-\nabla \tilde{\eta}_{e}(\vec{f}-\overrightarrow{\tilde{f}}),
\end{aligned}
$$

where

$$
\begin{array}{ll}
\tilde{\eta}_{e}=\eta_{e}(\tilde{n}, \bar{J}), & \tilde{q}_{e}=q_{e}(\tilde{n}, \bar{J}), \\
\vec{v}=(n, J)^{T}, & \vec{f}=\left(J, \frac{J^{2}}{n}+p(n)\right)^{T} .
\end{array}
$$

The following theorem is our main result.

Theorem 2: Let $(\tilde{n}, \bar{J}, \tilde{E})$ be given in Theorem 1. Let $(n, J, E)(x, t)$ be any weak entropy solution of (1), (3)-(5) satisfying H1) such that $y(x, 0) \in$ $L^{2}(\mathbf{R}), \int_{-\infty}^{\infty} \eta_{*}(x, 0) d x<\infty$, then there exists a positive constant $\delta$ such that if

$$
\bar{J} \leq \delta
$$

then

$$
\int_{-\infty}^{\infty}\left(y_{t}^{2}+y_{x}^{2}+y^{2}\right) d x \leq C e^{-\tilde{C} t} \int_{-\infty}^{\infty}\left(\eta_{*}(x, 0)+y^{2}(x, 0)\right) d x
$$

holds for any $t>0$ and some positive constants $C$ and $\tilde{C}$.

Proof: First, from (17) and (18) we have the following equation on $y$

$$
y_{t t}+\left(\frac{J^{2}}{n}-\frac{\bar{J}^{2}}{\tilde{n}}\right)_{x}+(p(n)-p(\tilde{n}))_{x}+y_{t}=-\tilde{n} y-\tilde{E} y_{x}+y y_{x} .
$$


Multiplying $y$ with $(22)$ and integrating over $(-\infty,+\infty)$, we get

$$
\begin{aligned}
& \left.\frac{d}{d t} \int_{-\infty}^{\infty}\left(y y_{t}+\frac{1}{2} y^{2}\right) d x+\int_{-\infty}^{\infty}(p(n)-p(\tilde{n}))(n-\tilde{n})+\left(\tilde{n}-\frac{\tilde{E}_{x}}{2}\right) y^{2}\right) d x \\
& \quad \leq \int_{-\infty}^{\infty} y_{t}^{2} d x+\int_{-\infty}^{\infty}\left(\frac{J^{2}}{n}-\frac{\bar{J}^{2}}{\tilde{n}}\right) y_{x} d x .
\end{aligned}
$$

We now note that

$$
\tilde{n}-\frac{\tilde{E}_{x}}{2}=\frac{1}{2}(\tilde{n}+b(x)) .
$$

Thanks to (14), the Lemma 3.1 of [6] says that

$$
(p(n)-p(\tilde{n}))(n-\tilde{n})=O(1)(n-\tilde{n})^{2}=O(1) y_{x}^{2} .
$$

Therefore, we conclude from (23) that there exists a positive constant $C_{2}$ such that

$$
\begin{gathered}
\frac{d}{d t} \int_{-\infty}^{\infty}\left(y y_{t}+\frac{1}{2} y^{2}\right) d x+C_{2} \int_{-\infty}^{\infty}\left(y_{x}^{2}+y^{2}\right) d x \\
\leq \int_{-\infty}^{\infty} y_{t}^{2} d x+\int_{-\infty}^{\infty}\left(\frac{J^{2}}{n}-\frac{\bar{J}^{2}}{\tilde{n}}\right) y_{x} d x .
\end{gathered}
$$

As our solution has no further regularity, standard energy estimates such as (26) do not generalize to higher order. Following the ideas introduced in [5] and [6], we will now explore the entropy dissipation. In view of the definition of $\eta_{*}$ and $q_{*}$ in (19), we substitute them into the entropy inequality (7) to obtain

$$
\begin{aligned}
0 \geq \eta_{e t}+ & q_{e x}-\frac{J}{n}(n E-J) \\
=\eta_{* t} & +q_{* x}+\nabla \tilde{\eta}_{e}(\vec{v}-\overrightarrow{\tilde{v}})_{t}+\nabla \tilde{\eta}_{e}(\vec{f}-\overrightarrow{\tilde{f}})_{x} \\
& \quad+\tilde{q}_{e x}+\left(\nabla \tilde{\eta}_{e}\right)_{x}(\vec{f}-\overrightarrow{\tilde{f}})-\frac{J}{n}(n E-J) .
\end{aligned}
$$

We now simplify the terms in (27). Using the equations (17), (18) and (22), we observe that

$$
\nabla \tilde{\eta}_{e}(\vec{v}-\overrightarrow{\tilde{v}})_{t}+\nabla \tilde{\eta}_{e}(\vec{f}-\overrightarrow{\tilde{f}})_{x}=\frac{\bar{J}}{\tilde{n}}\left(n E-\tilde{n} \tilde{E}-y_{t}\right),
$$

and

$$
\begin{aligned}
& \tilde{q}_{e x}+\left(\nabla \tilde{\eta}_{e}\right)_{x}(\vec{f}-\overrightarrow{\tilde{f}}) \\
& =\left[\gamma \tilde{n}^{\gamma-2} J+\frac{\bar{J}^{2}}{\tilde{n}^{3}} J-2 \frac{\bar{J}^{3}}{\tilde{n}^{3}}-\frac{\bar{J}}{\tilde{n}^{2}}\left(\frac{J^{2}}{n}-\frac{\bar{J}^{2}}{\tilde{n}}+p(n)-p(\tilde{n})\right)\right] \tilde{n}_{x} .
\end{aligned}
$$


We thus have from (27) that

$$
\begin{gathered}
\eta_{* t}+q_{* x} \leq J E-\frac{J^{2}}{n}-\frac{\bar{J}}{\tilde{n}}\left(n E-\tilde{n} \tilde{E}-y_{t}\right) \\
-\left[\tilde{q}_{e x}+\left(\nabla \tilde{\eta}_{e}\right)_{x}(\vec{f}-\overrightarrow{\tilde{f}})\right] .
\end{gathered}
$$

For further simplification is necessary, we note that

$$
\tilde{E}=\frac{1}{\tilde{n}}\left(\frac{\bar{J}^{2}}{\tilde{n}}+p(\tilde{n})\right)_{x}+\frac{\bar{J}}{\tilde{n}}=\gamma \tilde{n}^{\gamma-2} \tilde{n}_{x}-\frac{\bar{J}^{2}}{\tilde{n}^{3}} \tilde{n}_{x}+\frac{\bar{J}}{\tilde{n}},
$$

then

$$
\begin{aligned}
J E & =J \tilde{E}-y y_{t}-y \bar{J} \\
& =-\frac{\bar{J}^{2}}{\tilde{n}^{3}} J \tilde{n}_{x}+\gamma \tilde{n}^{\gamma-2} J \tilde{n}_{x}+\frac{\bar{J}}{\tilde{n}} y_{t}+\frac{\bar{J}^{2}}{\tilde{n}}-y y_{t}-y \bar{J} .
\end{aligned}
$$

On the other hand, we have

$$
\begin{gathered}
-\frac{\bar{J}}{\tilde{n}}\left(n E-\tilde{n} \tilde{E}-y_{t}\right)=-\frac{\bar{J}}{\tilde{n}}\left(y y_{x}-\tilde{E} y_{x}-y \tilde{n}-y_{t}\right) \\
=-\frac{\bar{J}^{3}}{\tilde{n}^{4}} y_{x} \tilde{n}_{x}+\gamma \tilde{n}^{\gamma-3} \bar{J} y_{x} \tilde{n}_{x}+\frac{\bar{J}^{2}}{\tilde{n}^{2}} y_{x}-\frac{\bar{J}}{\tilde{n}} y y_{x}+\bar{J} y+\frac{\bar{J}}{\tilde{n}} y_{t} .
\end{gathered}
$$

From (29)-(32), manipulating the terms properly, we conclude that

$$
\left(\eta_{*}+\frac{1}{2} y^{2}\right)_{t}+q_{* x}+Q_{1} \leq \frac{\bar{J} \tilde{n}_{x}}{\tilde{n}^{2}}\left(Q_{1}+Q_{2}+\frac{1}{2} y^{2}\right)-\left(\frac{\bar{J}}{2 \tilde{n}} y^{2}\right)_{x}
$$

where

$$
Q_{1}=\frac{J^{2}}{n}-\frac{\bar{J}^{2}}{\tilde{n}}-\frac{2 \bar{J}}{\tilde{n}} y_{t}-\frac{\bar{J}^{2}}{\tilde{n}^{2}} y_{x}, \quad Q_{2}=n^{\gamma}-\tilde{n}^{\gamma}+\gamma \tilde{n}^{\gamma-1} y_{x}
$$

Clearly, $Q_{1}$ is the quadratic remainder of the Taylor expansion of $\frac{J^{2}}{n}$ around $\bar{J}$ and $\tilde{n}$, while $Q_{2}$ is the one for pressure. Furthermore, $Q_{2}=0$ for $\gamma=1$. Due to the convexity, we remark that both $Q_{1}$ and $Q_{2}$ are non-negative.

Integrating (33) over $(-\infty,+\infty)$, we get

$$
\frac{d}{d t} \int_{-\infty}^{\infty}\left(\eta_{*}+\frac{1}{2} y^{2}\right) d x+\int_{-\infty}^{\infty} Q_{1} d x \leq \int_{-\infty}^{\infty} \frac{\bar{J} \tilde{n}_{x}}{\tilde{n}^{2}}\left(Q_{1}+Q_{2}+\frac{1}{2} y^{2}\right) d x
$$


Let $\Lambda=\max \left\{b^{*}, C_{0}\right\}$, where $C_{0}$ is given in $\mathbf{H 1}$ ). We now multiply (35) by $\lambda=2 \Lambda+1$, add the results to (23) to obtain

$$
\frac{d}{d t} \int_{-\infty}^{\infty} F_{1} d x+\int_{-\infty}^{\infty}\left(F_{2}+F_{3}+F_{4}\right) d x \leq 0
$$

where

$$
\begin{aligned}
& F_{1}=\lambda \eta_{*}+\frac{1}{2}(1+\lambda) y^{2}+y y_{t}, \\
& F_{2}=\lambda\left(1-\frac{\tilde{J}_{x}}{\tilde{n}^{2}}\right) Q_{1}-y_{t}^{2}-\left(\frac{J^{2}}{n}-\frac{\bar{J}^{2}}{\tilde{n}}\right) y_{x}, \\
& F_{3}=(p(n)-p(\tilde{n}))(n-\tilde{n})-\lambda \frac{\tilde{n}_{x}}{\tilde{n}^{2}} Q_{2}, \\
& F_{4}=\left[\left(\tilde{n}-\frac{\tilde{E}_{x}}{2}\right)-\frac{1}{2} \lambda \frac{\bar{J} \tilde{n}_{x}}{\tilde{n}^{2}}\right] y^{2} .
\end{aligned}
$$

By Theorem 1, we know there is $C_{3}>0$ such that

$$
\frac{\bar{J} \tilde{n}_{x}}{\tilde{n}^{2}} \leq C_{3} \delta
$$

For $F_{4}$, it is clear from (14) and (24) that

$$
F_{4} \geq \frac{1}{2}\left(2 b_{*}-\lambda C_{3} \delta\right) y^{2}
$$

When $\gamma=1, Q_{2}=0$, and thus $F_{3}=y_{x}^{2}$. When $\gamma>1$, Lemma 5.2 of [15] states that there exits a positive $C_{4}$ such that

$$
Q_{2} \leq C_{4}(p(n)-p(\tilde{n}))(n-\tilde{n}),
$$

and therefore

$$
F_{3} \geq\left(1-C_{3} C_{4} \lambda \delta\right)(p(n)-p(\tilde{n}))(n-\tilde{n}) .
$$

We now treat $F_{2}$. From the definition of $Q_{1}$ in $(34)$, we know that

$$
\left(\frac{J^{2}}{n}-\frac{\bar{J}^{2}}{\tilde{n}}\right) y_{x}=y_{x} Q_{1}+\frac{2 \bar{J}}{\tilde{n}} y_{t} y_{x}+\frac{\bar{J}^{2}}{\tilde{n}^{2}} y_{x}^{2} .
$$

On the other hand,

$$
\begin{aligned}
Q_{1} & =\frac{J^{2}}{n}-\frac{J^{2}}{\tilde{n}}+\frac{J^{2}}{\tilde{n}}-\frac{\bar{J}^{2}}{\tilde{n}}-\frac{2 \bar{J}}{\tilde{n}} y_{t}-\frac{\bar{J}^{2}}{\tilde{n}^{2}} y_{x} \\
& =\frac{y_{t}^{2}}{\tilde{n}}+\frac{y_{x}}{\tilde{n}}\left(\frac{J^{2}}{n}-\frac{J^{2}}{\tilde{n}}\right) \\
& =\frac{y_{t}^{2}}{\tilde{n}}+\frac{y_{x}}{\tilde{n}}\left(Q_{1}+\frac{2 \bar{J}}{\tilde{n}} y_{t}+\frac{\bar{J}^{2}}{\tilde{n}^{2}} y_{x}\right),
\end{aligned}
$$


from which we have

$$
\frac{n}{\tilde{n}} Q_{1}=\frac{y_{t}^{2}}{\tilde{n}}+\frac{2 \bar{J}}{\tilde{n}^{2}} y_{t} y_{x}+\frac{\bar{J}^{2}}{\tilde{n}^{3}} y_{x}^{2}
$$

Then we get

$$
\left\{\begin{aligned}
Q_{1} & \geq \frac{y_{t}^{2}}{\tilde{n}}+\frac{2 \bar{J}}{\tilde{n}^{2}} y_{t} y_{x}, \quad n \leq \tilde{n} ; \\
Q_{1} & \geq \frac{\tilde{n}}{n}\left(\frac{y_{t}^{2}}{\tilde{n}}+\frac{2 \bar{J}}{\tilde{n}^{2}} y_{t} y_{x}\right), \quad n>\tilde{n} .
\end{aligned}\right.
$$

We are now able to give a good estimate on $F_{2}$. From (40) and (42), and the fact that $\left|y_{x}\right| \leq \Lambda$, we have, for some positive constants $C_{5}$ and $C_{6}$, that

$$
\begin{aligned}
F_{2} & \geq\left[\lambda\left(1-C_{3} \delta\right)-y_{x}\right] Q_{1}-y_{t}^{2}-\frac{2 \bar{J}}{\tilde{n}} y_{t} y_{x}-\frac{\bar{J}^{2}}{\tilde{n}^{2}} y_{x}^{2} \\
& \geq C_{5} Q_{1}-C_{6} \delta y_{x}^{2}
\end{aligned}
$$

provided $C_{3} \lambda \delta<\frac{1}{2}$, this is achieved if $\delta<\frac{1}{2}\left(C_{3} \lambda\right)^{-1}$. Therefore, for suitably small $\delta$, we have from (38), (39) and (43) that there is $C_{7}>0$ such that

$$
F_{2}+F_{3}+F_{4} \geq C_{7}\left(Q_{1}+y_{x}^{2}+y^{2}\right) .
$$

We now turn to $F_{1}$. It is easy to see that

$$
\eta_{*}=\frac{Q_{1}}{2}+\frac{1}{\gamma-1} Q_{2}, \quad \gamma>1 ; \quad \eta_{*}=\frac{Q_{1}}{2}+Q_{3}, \quad \gamma=1,
$$

where

$$
Q_{3}=n \ln n-\tilde{n} \ln \tilde{n}-(\ln \tilde{n}+1)(n-\tilde{n}) .
$$

From Lemma 3.1 of [6], we know that there is $C_{8}>0$ such that

$$
Q_{2} \geq C_{8} y_{x}^{2}
$$

We now claim that there are $C_{9}>0$ and $C_{10}>0$ such that

$$
C_{9} y_{x}^{2} \leq Q_{3} \leq C_{10} y_{x}^{2}
$$

Indeed, $Q_{3}$ is the quadratic remainder of the Taylor expansion of the convex function $n \ln n$ about $\tilde{n} \geq b_{*}>0$. (48) is easily proven using the strict convexity of $n \ln n$ and the bound of $n$. Therefore, we conclude from (37), 
(42), (45)-(48) and the smallness of $\delta$ that there are $C_{11}>0$ and $C_{12}>0$ such that

$$
C_{11}\left(y^{2}+y_{x}^{2}+y_{t}^{2}\right) \leq C_{12}\left(y^{2}+y_{x}^{2}+Q_{1}\right) \leq F_{1} \leq C_{13}\left(y^{2}+y_{x}^{2}+Q_{1}\right) .
$$

Hence, (36), (44) and (49) imply that

$$
\frac{d}{d t} \int_{-\infty}^{\infty} F_{1} d x+\frac{C_{7}}{C_{13}} \int_{-\infty}^{\infty} F_{1} d x \leq 0
$$

which together with (49) yields the decay estimate (21). This completes the proof of Theorem 2.

\section{Conclusion Remarks}

In this section, we make some remarks on our assumptions, results and proofs.

1) In the proof of Theorem 2 , we used the fact that $y( \pm \infty, t)=0$. The initial assumption does imply that $y( \pm \infty, 0)=0$. Therefore, $(21)$ and standard continuity argument do verify this fact.

2) We also remark that the smallness assumption on $\bar{J}$ also ensures H2), that guarantees the subsonic condition. However, it is clear that the proof of Theorem 2 is still valid if we request small background velocity $\frac{\bar{J}}{b_{*}}$ instead. Furthermore, $\delta$ does not have to be arbitrary small in our Theorem 2. It is clear from our proof that one may determine a constant upper bound for $\delta$ depending on $b_{*}, b^{*}, C_{0}$ and certain convex functions appear in the proof. This will leads to a tedious elementary calculation which is not the main purpose of this paper. It is not clear whether the statement (21) is true without any restriction on the amplitude of $\bar{J}$ or background velocity.

3) The uniform upper bound on density is very important in our proof. We remark that the uniform bound (8) on $n$ is still an open problem for $L^{\infty}$ weak entropy solutions to system (1), although it seems natural from physical point of view. The bounds obtained in [13] or [16] grow in time. However, for the piecewise smooth solutions constructed in [9], the uniform bound (8) is verified. Also, the sub-critical global smooth solutions constructed in [17] 
have uniform upper bound on $n$. Therefore, our Theorem 2 is valid for the solutions obtained by [9] and [17].

Acknowledges: The research of F. Huang is also partially supported by NSFC grant (No. 10471138), NSFC-NSAF grant (No. 10676037) and 973 program of China (No. 2006CB805902). The research of R. Pan is partially supported by NSF grant through DMS- 0505515.

\section{References}

[1] G. Ali, Global existence of smooth solutions of the $N$-dimensional EulerPoisson model, SIAM J. Math. Anal., 35 (2003), 389-422.

[2] G. Q. Chen and D. H. Wang, Formation of singularity in compressible Euler-Poisson fluids with heat diffusion and damping relaxation, J. Differential Equations., 144 (1998), 44-65.

[3] I. Gamba, Stationary transonic solutions of a one-dimensional hydrodynamic model for semiconductor, Comm. Partial Differential Equations., 17 (1992), 553-577.

[4] Y. Guo and W. Strauss, Stability of semiconductor states with insulating and contact boundary conditions, Arch. Rational Mech. Anal., 179 (2005), 1-30.

[5] F. Huang, P. Marcati and R. Pan Convergence to Barenblatt solution for the compressible Euler equations with damping and vacuum, Arch. Ration. Mech. Anal., 176(2005), 1-24.

[6] F. Huang, R. Pan, Convergence rate for compressible Euler Equations with damping and vacuum, Arch. Rational Mech. Anal., 166 (2003), 359-376.

[7] H. Hattori and C. Zhu, Asymptotic behavior of the solutions to a nonisentropic hydrodynamic model of semiconductors, J. Differential Equations, 144 (1998), 353-389.

[8] H. L. Li, P. Markowich, and M. Mei, Asymptotic behavior of solutions of the hydrodynamic model of semiconductors, Proc. Roy. Soc. Edinburgh Sect. A., 132(2002), 359-378. 
[9] H. L. Li, P. Markowich, and M. Mei, Asymptotic behavior of subsonic entropy solutions of isentropic Euler-Poisson equations, Quart. Appl. Math., 60(2002), 773-796.

[10] T. Luo, R. Natalini, and Z. P. Xin, Large time behavior of the solutions to a hydrodynamic model for semiconsuctors, SIAM J. Appl. Math., 59 (1998), 810-830.

[11] P. Marcati and M. Mei, Asymptotic convergence to steady-state solutions of the initial boundary value problem to a hydrodynamic model for semiconductors, unpublished preprint.

[12] A. Matsumura and T. Murakami, Asymptotic behavior of solutions for a fluid dynamical model of semiconductor equation Research Institute of Mathematical Sciences, Kokyuroku, 1495 (2005), 60-70.

[13] P. Marcati and R. Natalini, Weak solutions to a hydrodynamic model for semiconductors: the Cauchy problem, Proc. Roy. Soc. Edinburgh Sect. A., 125(1995), 115-131.

[14] P. Markowich, C. Ringhofer and C. Schmeiser, Semiconductors equations, Springer-Verlag, 1990.

[15] R. Pan and K. Zhao, Initial Boundary value problem For compressible Euler equations with damping, Preprint, 2007.

[16] F. Poupaud. M. Rascle and J. P. Vila, Global solutions to the isothermal Euler-Poisson system with arbitrarily large data, J. Differential Equations., 123(1995), 93-121.

[17] E. Tadmor and D. Wei, On the global regularity of sub-critical EulerPoisson equations with pressure, Preprint, 2007.

[18] D. H. Wang and Z. J. Wang, Large BV solutions to the compressible isothermal Euler-Poisson equations with spherical symmetry, Nonlinearity, 19(2006), 1985-2004. 